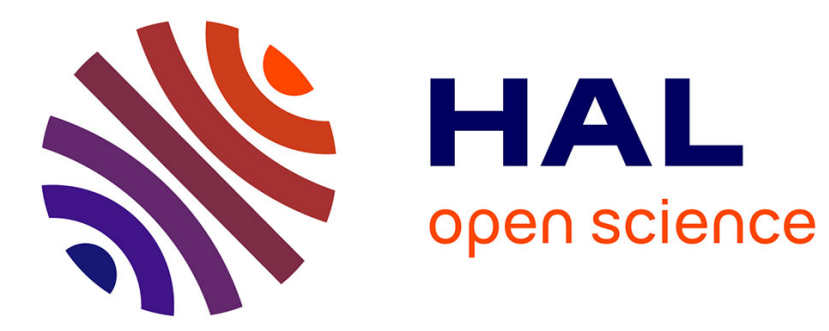

\title{
Oscillating singularities in locally self-similar functions
}

\author{
Alain Arneodo, Emmanuel Bacry, Jean-François Muzy
}

\section{To cite this version:}

Alain Arneodo, Emmanuel Bacry, Jean-François Muzy. Oscillating singularities in locally self-similar functions. Physical Review Letters, 1995, 74 (24), pp.4823-4826. 10.1103/PhysRevLett.74.4823. hal-01557134

\section{HAL Id: hal-01557134 \\ https://hal.science/hal-01557134}

Submitted on 5 Jul 2017

HAL is a multi-disciplinary open access archive for the deposit and dissemination of scientific research documents, whether they are published or not. The documents may come from teaching and research institutions in France or abroad, or from public or private research centers.
L'archive ouverte pluridisciplinaire HAL, est destinée au dépôt et à la diffusion de documents scientifiques de niveau recherche, publiés ou non, émanant des établissements d'enseignement et de recherche français ou étrangers, des laboratoires publics ou privés. 


\title{
Oscillating Singularities in Locally Self-Similar Functions
}

\author{
A. Arneodo, ${ }^{1}$ E. Bacry, ${ }^{2}$ and J. F. Muzy ${ }^{1}$ \\ ${ }^{1}$ Centre de Recherche Paul Pascal, Avenue Schweitzer, 33600 Pessac, France \\ ${ }^{2}$ Unité Fondamentale de Recherche de Mathématiques, Université de Paris VII, Tour 45-55, 2 Place Jussieu, \\ 75251 Paris Cedex 05, France
}

\begin{abstract}
Singularities induced by oscillating behavior are analyzed using the wavelet transform. We define two local exponents which allow us to characterize both the singularity strength (Hölder exponent) and the instantaneous frequency of the oscillations. Such oscillating singularities are shown to appear generically in local self-similar functions which are invariant under a nonhyperbolic mapping. We illustrate our results on both isolated singularities and nonisolated singularities appearing in fractal signals generated by nonhyperbolic iterative function systems.
\end{abstract}

During the past few years, there has been increasing interest in the study of irregular objects [1]. Fractal and multifractal concepts have been developed to describe the singular nature of such distributions through their scale invariance (self-similarity) properties $[1,2]$. They have been applied successfully in various physical situations ranging from the characterization of ramified patterns observed in growth experiments [3] to the study of the statistics of the velocity and the dissipation fields in fully developed turbulence [4,5]. In the latter context, some authors [6] advocate the use of models of eddy structures involving "spiral-type" behavior of the generic form

$f(x)=\left|x-x_{0}\right|^{\gamma} \sin \left(\frac{2 \pi}{\left|x-x_{0}\right|^{\beta}}\right) \quad(\beta>0, \gamma>-1)$.

Unfortunately, the multifractal formalism introduced in Ref. [2(a)] does not take into consideration these oscillating singular behaviors. The aim of this Letter is to study such locally accumulating oscillating behavior using a tool that has proven particularly powerful for analyzing singular functions, namely, the wavelet transform (WT) [7]. Within the general framework of WT analysis, we introduce two local exponents $\alpha\left(x_{0}\right)$ and $\varphi\left(x_{0}\right)$. The exponent $\alpha\left(x_{0}\right)$ can be seen as a generalization of the local singularity exponent commonly used in the multifractal description, whereas the exponent $\varphi\left(x_{0}\right)$ accounts for the behavior of the "instantaneous frequency." For singular behavior induced by "fast oscillations" [e.g., Eq. (1)], we show that $\varphi\left(x_{0}\right)$ plays an important role in the characterization of the strength of the singularity. This Letter is mainly devoted to the calculation of $\alpha$ and $\varphi$ for locally self-similar functions. We demonstrate that oscillating (nonoscillating) singularities are generically associated to invariance properties under nonhyperbolic (hyperbolic) mappings. We illustrate our purpose on particular examples including the fractal coding function recently introduced by Gutzwiller and Mandelbrot [8].

The WT of a signal permits an analysis both in physical space and in scale space. It consists in decomposing a signal in terms of wavelets which are constructed from one single function, the analyzing wavelet $\psi$, by means of dilations and translations. The WT of a function $f(x)$ is defined as [7]

$$
T_{\psi}(b, a)=\frac{1}{a} \int_{-\infty}^{+\infty} f(x) \psi\left(\frac{x-b}{a}\right) d x,
$$

where $b$ is a space parameter $(b \in R$ ) and $a$ a scale parameter $\left(a \in R^{+*}\right)$. The analyzing wavelet $\psi(x)$ is chosen well localized around $x=0$ and with a vanishing integral so that a large value of $\left|T_{\psi}(b, a)\right|$ corresponds to a large variation of $f(x)$ over a distance $a$ from the point $x=b$. Thus, as proved by Mallat and Hwang [9], if $f(x)$ is singular at $x=x_{0}$, then, at any scale $a$ (arbitrarily small), the function $\left|T_{\psi}(\cdot, a)\right|$ is locally maximum at a point $b(a)$ in the neighborhood of $x_{0}$. The points $(b(a), a)$ in the space-scale half plane are generally referred to as WT modulus maxima (WTMM); they are lying on connected curves called maxima lines. These lines generally converge to the points where $f$ is singular $[9,10]$ [i.e., $b(a) \rightarrow x_{0}$ when $a \rightarrow 0^{+}$]. Moreover, provided some first moments of $\psi$ are zero, one can recover [9-11] the Hölder exponent $h\left(x_{0}\right)$ of $f$ at the point $x_{0}$ by studying the power-law behavior of the WT along a maxima line converging to $x_{0}$ :

$$
T_{\psi}((b(a), a)) \sim a^{h\left(x_{0}\right)}, \quad a \rightarrow 0^{+} .
$$

Let us recall that this exponent characterizes the singularity strength of $f(x)$ at the point $x=x_{0}$ :

$$
\begin{aligned}
f\left(x_{0}+l\right)= & f\left(x_{0}\right)+l f^{(1)}\left(x_{0}\right)+\cdots+\left(l^{n} / n !\right) f^{(n)}\left(x_{0}\right) \\
& +O\left(|l|^{h\left(x_{0}\right)}\right),
\end{aligned}
$$

where $n<h\left(x_{0}\right)<n+1$ and $f^{(k)}$ is the $k$ th derivative of $f$.

However, Eq. (3) is not relevant in the case of oscillating singularities [9], i.e., such that the Hölder exponent is increased by more than 1 when the function is integrated (this is generally induced by the presence of an 
infinite number of accumulating oscillations in any neighborhood of the singularity). Let us illustrate this irrelevance on the particular class of "infinitely oscillating" functions defined in Eq. (1). These functions are singular at $x_{0}$ and correspond, according to the definition [Eq. (4)], to the Hölder exponents $h\left(x_{0}\right)=\gamma$. The graph of such a function is displayed in Fig. 1(a). As one can see in Fig. 1(b), the WTMM of this function are lying on an infinite (countable) number of maxima lines $\left\{l_{n}\right\}_{n \in \mathbb{Z}}$ appearing at smaller and smaller scales. Each maxima line corresponds to an oscillation in the signal, and the scale at which it appears is proportional to the distance between two successive maxima lines. This distance can be seen as an "instantaneous period." It is clear that the WT along any maxima line, for $a$ small enough, will not account for the singularity behavior of $f$ at $x_{0}=0$ since none of these lines converge towards the point $x_{0}=0$. Thus, instead of following a maxima line, as in Eq. (3), one must "jump" from one line to another in order to converge towards $x_{0}$ when going from large to small scales. Let us consider the maxima $\left(b_{n}, a_{n}\right)$ which correspond to the greatest value of $\left|T_{\psi}\right|$ on each line $l_{n}$ (if it is not unique we consider the one
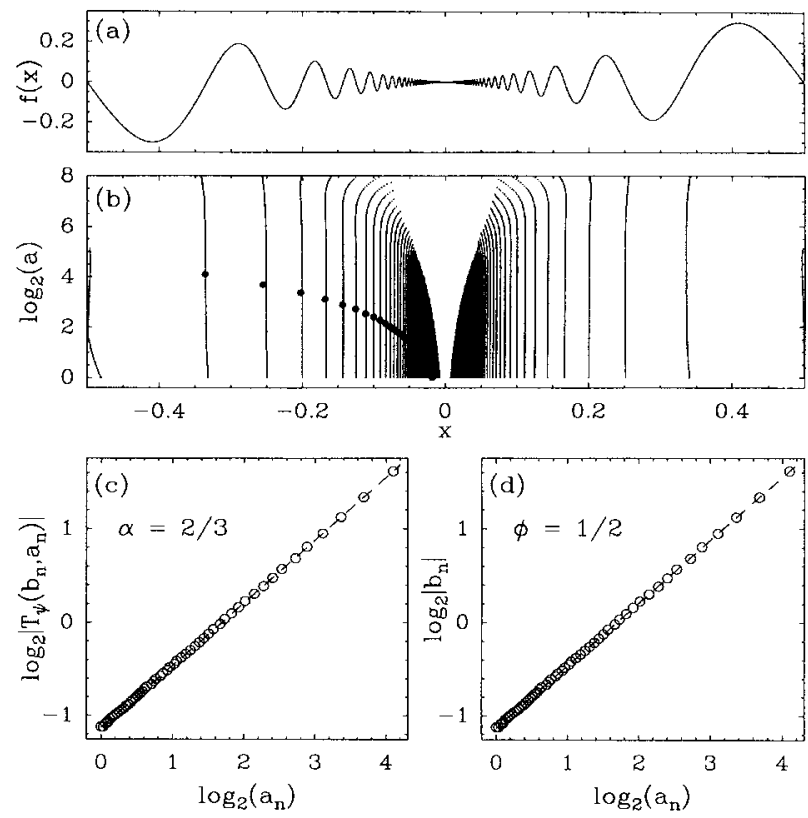

FIG. 1. Detection of the local exponents $\alpha\left(x_{0}\right)$ and $\varphi\left(x_{0}\right)$ associated to an oscillating singularity. (a) Graph of the function $f(x)=|x|^{\gamma} \sin \left(2 \pi /|x|^{\beta}\right)$ for $\gamma=\frac{4}{3}$ and $\beta=1$. The point $x_{0}=0$ corresponds to an oscillating singularity of $f$. (b) WT skeleton showing the positions of the modulus maxima for the signal in (a). The analyzing wavelet $\psi$ is the first derivative of the Gaussian function. The maxima are lying on maxima lines. Along each line $l_{n}$, the symbol $(\bullet)$ marks the point $\left(a_{n}, b_{n}\right)$ where the WT is the greatest. (c) $\log _{2}\left|T \psi\left(b_{n}, a_{n}\right)\right|$ vs $\log _{2} a_{n}$. The slope provides an estimate of $\alpha\left(x_{0}\right)=\gamma /(\beta+$ 1). (d) $\log _{2}\left|b_{n}\right|$ vs $\log _{2} a_{n}$. The slope gives an estimate of $\varphi\left(x_{0}\right)=1 /(\beta+1)$. appearing at the largest scale). As shown in Fig. 1(b), $b_{n}$ goes to $x_{0}$ when $a_{n}$ goes to 0 . [It can be proven that the points $\left(b_{n}, a_{n}\right)$ belong to the "ridge" defined in Ref. [12] as the instantaneous frequency curve in the $(b, a)$ spacescale half plane.] Moreover, as illustrated in Fig. 1(c), one can prove that, along this sequence, the WT modulus follows a power-law scaling

$$
\left|T_{\psi}\left(b_{n}, a_{n}\right)\right| \sim a_{n}^{\alpha\left(x_{0}\right)},
$$

where $\alpha\left(x_{0}\right)=\gamma /(\beta+1)$. The so-obtained local exponent $\alpha\left(x_{0}\right)$ is smaller than the Hölder exponent $h\left(x_{0}\right)=\gamma$. It thus appears that, in order to extract $h\left(x_{0}\right)$, we must characterize the oscillations of $f(x)$, i.e., evaluate the exponent $\beta$. From $\alpha$ and $\beta$ we will then naturally recover $\gamma=h\left(x_{0}\right)$.

Since each of the maxima line corresponds to an oscillation in the signal, the abscissa $b_{n}$ behave like $\left|b_{n}-x_{0}\right| \sim n^{-1 / \beta}$. Moreover, the scale $a_{n}$ is of the order of the distance between this maxima line and the next one, i.e., $a_{n} \sim b_{n}-b_{n+1} \sim n^{-(\beta+1) / \beta}$. Thus the distance between the $n$th maxima line and the singularity $x_{0}$ scales like

$$
\left|b_{n}-x_{0}\right| \sim a_{n}^{\varphi\left(x_{0}\right)}, \quad n \rightarrow+\infty,
$$

where $\varphi\left(x_{0}\right)=1 /(\beta+1)$. As illustrated in Fig. 1(d), the exponent $\varphi\left(x_{0}\right)$ can thus be extracted from a simple log$\log$ plot of the distance $\left|b_{n}-x_{0}\right|$ vs the scale $a_{n}$. The Hölder exponent $h\left(x_{0}\right)$ can thus be recovered through the particularly simple relation

$$
h\left(x_{0}\right)=\alpha\left(x_{0}\right) / \varphi\left(x_{0}\right) .
$$

Let us note that Eqs. (5), (6), and (7) hold for a general class of analyzing wavelets including the successive derivatives of the Gaussian functions [2(c)].

For a general function $f$, we define the exponent $\alpha\left(x_{0}\right)$ as in Eq. (5) and $\varphi\left(x_{0}\right)$ as the minimum of $\varphi\left(x_{0}\right)$ [Eq. (6)] and 1. The so-defined local exponents allow us to characterize very precisely the strength and the nature of a singular behavior at a given point $x_{0}$. Indeed, for a very large class of functions, one can prove that the relation [Eq. (7)] still holds; i.e., the strength of the singularity at $x_{0}$ is directly measured by the ratio $\alpha\left(x_{0}\right) / \varphi\left(x_{0}\right)$. Moreover, $\varphi\left(x_{0}\right)=1$ indicates that $x_{0}$ corresponds to a nonoscillating singularity. In that case, we thus get $\alpha\left(x_{0}\right)=h\left(x_{0}\right)$; i.e., Eq. (3) holds and becomes the same as Eq. (5). On the other hand, a value of $\varphi\left(x_{0}\right)$ smaller than 1 corresponds to an oscillating singularity and, consequently, to an exponent $\alpha\left(x_{0}\right)$ smaller than $h\left(x_{0}\right)$. Equation (3) does not hold anymore. The smaller $\varphi\left(x_{0}\right)$ the more oscillating $f$ in the neighborhood of $x_{0}$. Moreover, one can prove that the derivative $f^{\prime}(x)=$ $d f / d x$ corresponds generically to the exponents $\alpha^{\prime}\left(x_{0}\right)=$ $\alpha\left(x_{0}\right)-1$ and $\varphi^{\prime}\left(x_{0}\right)=\varphi\left(x_{0}\right)$. The Hölder exponent of $f^{\prime}$ at $x_{0}$ can thus be derived from the one of $f$ using Eq. (7):

$$
h^{\prime}\left(x_{0}\right)=h\left(x_{0}\right)-1 / \varphi\left(x_{0}\right) .
$$


Hence, the more oscillating the signal the more irregular its derivative. Let us note that mathematicians have recently refined the notion of Hölder spaces by introducing a 2-microlocal spaces $[13,14]$ that allows a better characterization of the action of integration or differentiation operators in functional analysis. Our exponents $\alpha$ and $\varphi$ can actually be linked to the 2-microlocalization local exponents $s$ and $s^{\prime}$ through the relation $\alpha=s+s^{\prime}(1-\varphi)$.

Let us now consider the class of functions (or distributions) $f(x)$ which are locally self-similar around a given point $x_{0}=0$. We suppose that there exists an increasing function $F(x)$ with a fixed point $x_{0}$ which is stable under the iteration of $F$ and such that $f(x)=f(F(x)) / P(x)$, where $P(x)$ is some smooth weight function satisfying $0<P\left(x_{0}\right) \leq 1$. This invariance property of $f$ can be easily transposed in terms of self-similarity of its WT [15]:

$$
T_{\psi}(b, a) \simeq \frac{1}{P(b)} T\left(F(b), F^{\prime}(b) a\right),
$$

where $F^{\prime}(x)$ is the first derivative of $F(x)$. The same kind of relation naturally holds for the modulus maxima sequence $\left(b_{n}, a_{n}\right)$ corresponding to the greatest values of the WT along the maxima lines. By iterating this relation, one gets $b_{n}=F^{[n]}\left(b_{0}\right)$ and $a_{n}=\left(F^{[n]}\right)^{\prime}\left(b_{0}\right) a_{0}$, where $F^{[n]}$ is the $n$th iterate of $F$. Moreover $T_{n}=\left|T_{\psi}\left(b_{n}, a_{n}\right)\right| \sim$ $P\left(b_{0}\right) P\left(b_{1}\right) \cdots P\left(b_{n}\right)$. One can distinguish two cases.

(i) $F^{\prime}\left(x_{0}\right)<1$; i.e., $x_{0}$ is an hyperbolic fixed point. One can easily prove that $\left|b_{n}-x_{0}\right| \sim a_{n} \sim F^{\prime}\left(x_{0}\right)^{n}$ when $n \rightarrow$ $\infty$. One thus gets $\varphi\left(x_{0}\right)=1$; i.e., the singularity located at $x_{0}$ is a nonoscillating singularity and $h\left(x_{0}\right)=\alpha\left(x_{0}\right)$. The value of the exponent $\alpha\left(x_{0}\right)$ depends upon the expression of $P(x)$. In the case where $P\left(x_{0}\right)<1,\left[T_{n} \sim P\left(x_{0}\right)^{n}\right]$, we obtain $h\left(x_{0}\right)=\alpha\left(x_{0}\right)=-\ln (P) / \ln \left[F^{\prime}\left(x_{0}\right)\right]$.

(ii) $F^{\prime}\left(x_{0}\right)=1$; i.e., $x_{0}$ is a nonhyperbolic fixed point. Generically, in the neighborhood of $x_{0}$, one can write $F(x)=F\left(x_{0}\right)+x-x_{0}-C\left|x-x_{0}\right|^{r}+o\left[\left(x-x_{0}\right)^{r}\right](C>$ 0 and $r>1)$. Using the former expressions of $b_{n}$ and $a_{n}$, one gets $\left|b_{n}-x_{0}\right| \sim n^{1 /(1-r)}$ and $a_{n} \sim n^{r / 1-r}$. We thus obtain, from Eq. (6), $\varphi\left(x_{0}\right)=1 / r<1$; i.e., $x_{0}$ corresponds to an oscillating singularity. Again, the value of the exponent $\alpha\left(x_{0}\right)$ depends on the expression of $P(x)$. The case where $P\left(x_{0}\right)<1$ is degenerated in the sense that $\alpha\left(x_{0}\right)=+\infty$; i.e., $h\left(x_{0}\right)=+\infty ; f(x)$ is not singular at $x=x_{0}$, it is $C^{\infty}$ even though it is infinitely oscillating around $x_{0}$. However, in the same spirit as for SRB (SinaiRuelle-Bowen) measures [16], if we choose the weight function $P(x)=\left|F^{\prime}(x)\right|^{\eta}$ for $x$ in the neighborhood of $x_{0}$ ( $\eta$ is an arbitrary positive real number), then we get $T_{n} \sim\left[F^{[n]^{\prime}}\left(b_{0}\right)\right]^{\eta} T_{0}$ and thus $\alpha\left(x_{0}\right)=\eta . \quad f$ is singular at $x_{0}$ with an Hölder exponent $h\left(x_{0}\right)=r \eta$.

Oscillating singularities thus appear generically in locally self-similar functions invariant under nonhyperbolic mappings. The functions $x^{\gamma} \sin \left(2 \pi / x^{\beta}\right)$ [Eq. (1)] are actually good examples of such functions. Indeed, they are invariant under the mapping $F(x)=x /\left(1+x^{\beta}\right)^{1 / \beta}$ $\left[F^{\prime}(0)=1\right.$ and $\left.r=\beta+1\right]$ with $P(x) \sim F^{\prime}(x)^{\eta}$ [where $\eta=\gamma /(\beta+1)]$. According to the considerations just above, one easily recovers $\varphi(0)=1 / r=1 /(\beta+1)$ and $\alpha(0)=\eta=\gamma /(\beta+1)$. In Fig. 2(c), we have displayed a fractal measure which has been constructed on the interval $[0,1]$ by iterating the iterative function system (IFS) [17] shown in Fig. 2(a). Each branch of the IFS, $F_{1}(x)=x /(1+3 x)\left[F_{2}(x)=(x+1) / 2\right]$, is associated with the weight function $P_{1}(x)=\left|F_{1}^{\prime}(x)\right|^{1 / 3}\left[P_{2}(x)=\right.$ $\left.(1 / 2)^{1 / 3}\right]$. Each point of the support of the measure can
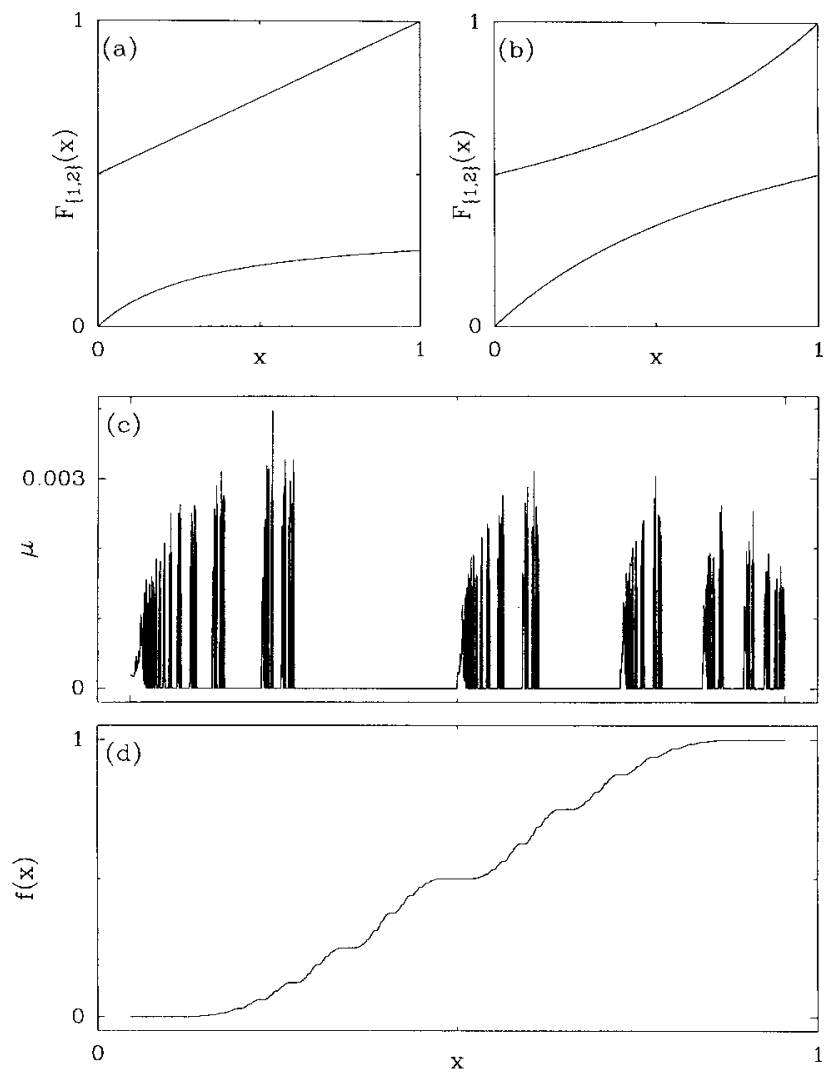

FIG. 2. Fractal distributions with oscillating behavior. (a) Two branch IFS, $F_{1}(x)=x /(1+3 x)$ and $F_{2}(x)=(x+1) / 2$, used to build the measure in (c). Only the second branch is hyperbolic $\left[F_{1}^{\prime}(0)=1, F_{2}^{\prime}(1)=\frac{1}{2}\right]$. (b) Two branch IFS, $F_{1}(x)=x /(1+x)$ and $F_{2}(x)=1 /(2-x)$, used to build the function in (d). Both branches are nonhyperbolic $\left[F_{1}^{\prime}(0)=\right.$ $\left.F_{2}^{\prime}(1)=1\right]$. (c) Fractal measure generated by the two branch IFS shown in (a) when using the respective weight functions $P_{\mathrm{i}}(x)=\left|F_{1}^{\prime}(x)\right|^{1 / 3}$ and $P_{2}(x)=(1 / 2)^{1 / 3}$. Any point of the support of the measure can be addressed through a symbolic sequence of 1 's and 2's. As $F_{1}$ is nonhyperbolic, any symbolic sequence which ends with an infinite number of 1 corresponds to an oscillating singularity $\left(\varphi=\frac{1}{2}\right)$. (d) Graph of the Gutzwiller-Mandelbrot function generated by the two branch nonhyperbolic IFS displayed in (b). Constant $(<1)$ weight functions are used; the oscillating behaviors are thus degenerated in the sense that $\alpha=h=+\infty$; i.e., the signal is not singular although infinitely oscillating. These behaviors are dense in $[0,1]$. All the other points correspond to nonoscillating singularities. 
be addressed by a symbolic sequence of 1 's and 2's, e.g., $x=0$ corresponds to the symbolic sequence $1111 \ldots$ whereas $x=1$ corresponds to $2222 \ldots$. As the first branch is nonhyperbolic, one can show that all the symbolic sequences which end with an infinite number of 1 's correspond to oscillating singularities. In the same way, the sequences ending with an infinite number of 2's correspond to nonoscillating singularities. Actually one can show that this is also the case for any periodic symbolic sequence which does not end by an infinite number of l's. Thus, in the neighborhood of any oscillating singularity, there exists an infinite number of both oscillating and nonoscillating singularities.

As the last example, let us consider the coding function introduced by Gutzwiller and Mandelbrot [8] in the context of chaotic Hamiltonian systems. It has been proven by Bessis and Mantica [18] that this function can be obtained using a 2-branch IFS [Fig. 2(b)] whose two branches are nonhyperbolic. The graph of this coding function is shown in Fig. 2(d). Nonoscillating $(\varphi=1$, e.g., periodic symbolic sequences) and oscillating behavior $(\varphi<1)$ are locally accumulating. However, the weight functions associated to the nonhyperbolic branches are strictly smaller than 1 . Thus, the oscillating behaviors are "degenerated" in the sense that they do not induce any singularity $(\alpha=h=+\infty)$. These "smooth" behaviors are responsible for the slow decay of the $f(\alpha)$ singularity spectrum estimated by Gutzwiller and Mandelbrot [8] using the classical multifractal formalism.

To summarize, we have thus defined two new local exponents which allow us to characterize very precisely both the nature and the strength of a singular behavior. These wavelet-based exponents [Eqs. (5) and (6)] can be seen as a generalization of the classical singularity exponent. They appear to be crucial for the understanding of the change of local regularity properties of a distribution when operating differentiation or integration. Let us note that the classical multifractal formalism [or its waveletbased generalization [2(c),5,10]] accounts only for the fluctuations of the scaling exponent defined in Eq. (3), which has no meaning when oscillating singularities are present. We have shown that such oscillating behaviors generically appear in fractal objects that are self-similar under nonhyperbolic mappings. These situations are commonly encountered in mathematics or physics; let us mention, for example, the famous Riemann-Weierstrass function $[11(b)]$ or the Farey-tree partitioning of rationals used to study the distribution of mode-locking intervals for critical circle maps [19]. A "grand-canonical" multifractal formalism that would account for the fluctuations of both exponents $\alpha$ and $\varphi$ would be of fundamental interest in this context.

Note added.--After completion of this work, we have been aware of a work by S. Jaffard and Y. Meyer [14] who introduced similar exponents in the context of "shirp analysis."

[1] B. Mandelbrot, The Fractal Geometry of Nature (Freeman, San Francisco, 1982).

[2] (a) T. C. Halsey, M. H. Jensen, L. P. Kadanoff, I. Procaccia, and B.I. Shraiman, Phys. Rev. A 33, 1141 (1986); (b) P. Collet, J. Lebowitz, and A. Porzio, J. Stat. Phys. 47, 609 (1987); (c) J. F. Muzy, E. Bacry, and A. Arneodo, Int. J. Bifurcation and Chaos 4, 245 (1994).

[3] T. Vicsek, Fractal Growth Phenomena (World Scientific, Singapore, 1992).

[4] U. Frisch and G. Parisi, in Turbulence and Predictability in Geophysical Fluid Dynamics and Climate Dynamics, edited by M. Ghil, R. Benzi, and G. Parisi (North-Holland, Amsterdam, 1985), p. 84; G. Paladin and A. Vulpiani, Phys. Rep. 156, 147 (1987); C. Meneveau and K. R. Sreenivasan, J. Fluid Mech. 224, 429 (1991).

[5] J. F. Muzy, E. Bacry, and A. Arneodo, Phys. Rev. Lett. 67, 3515 (1991).

[6] T. S. Lundgren, Phys. Fluids 25, 2193 (1982); H. K. Moffat, Turbulence and Chaotic Phenomena (North-Holland, Amsterdam, 1983); J.C.R. Hunt and J.C. Vassilicos, Proc. R. Soc. London A 434, 183 (1991).

[7] Wavelets, edited by J.M. Combes, A. Grossman, and P. Tchamitchian (Springer-Verlag, Berlin, 1989); Y. Meyer, Ondelettes (Hermann, Paris, 1990); I. Daubechies, Ten Lectures on Wavelets (S.I.A.M., Philadelphia, 1992).

[8] M. C. Gutzwiller and B. B. Mandelbrot, Phys. Rev. Lett. 60, 673 (1988).

[9] S. Mallet and W. L. Hwang, IEEE Trans. Inf. Theory 38 , 617 (1992)

[10] E. Bacry, J. F. Muzy, and A. Arneodo, J. Stat. Phys. 70, 635 (1993).

[11] (a) S. Jaffard, C. R. Acad. Sci. Paris I 308, 79 (1989); (b) M. Holschneider and P. Tchamitchian, in Les Ondelettes en 1989, edited by P.G. Lemarié (SpringerVerlag, Berlin, 1990), p. 102.

[12] N. Delprat, B. Escudié, P. Guillemain, R. KrolandMartinet, $\mathrm{Ph}$. Tchamitchian, and $\mathrm{B}$. Torrésani, IEEE Trans. Inf. Theory 38, 644 (1992).

[13] J.M. Bony, in Probabilistic Methods in Mathematical Physics: Proceedings of the Taniguchi International Symposium, 1985, Katata and Kyoto, edited by Ito Kiyosi and Ikeda Nobuyuki (Academic Press, Orlando, 1987), p. 11.

[14] S. Jaffard and Y. Meyer, "Pointwise behavior of functions" (to be published).

[15] A. Arneodo, E. Bacry, and J. F. Muzy, Europhys. Lett. 25, 479 (1994)

[16] J.P. Eckmann and D. Ruelle, Rev. Mod. Phys. 57, 617 (1985); T. Tél, Z. Naturforsch. A 48, 1154 (1988).

[17] C. R. Handy and G. Mantica, Physica (Amsterdam) 43D, 17 (1990).

[18] D. Bessis and G. Mantica, Phys. Rev. Lett. 66, 2939 (1991).

[19] R. Artuso and P. Cvitanović, Phys. Rev. A 39, 268 (1989). 